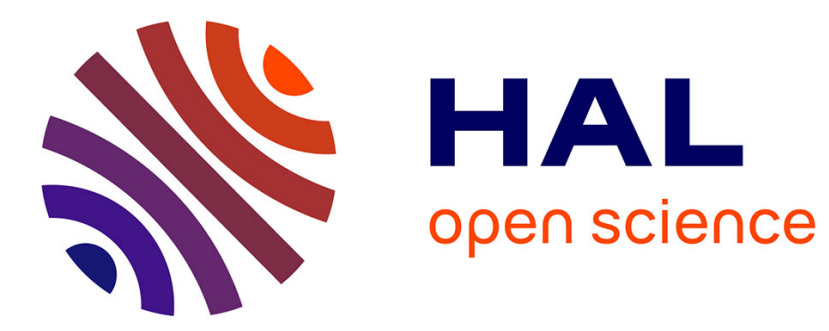

\title{
Les fouilles de Marloux (Saône-et-Loire) 1944-1945
}

\author{
Louis Armand-Calliat
}

\section{To cite this version:}

Louis Armand-Calliat. Les fouilles de Marloux (Saône-et-Loire) 1944-1945. Gallia - Fouilles et monuments archéologiques en France métropolitaine, 1947, 5 (2), pp.417-426. 10.3406/galia.1947.2049 . hal-01920955

\section{HAL Id: hal-01920955 \\ https://hal.science/hal-01920955}

Submitted on 27 Feb 2020

HAL is a multi-disciplinary open access archive for the deposit and dissemination of scientific research documents, whether they are published or not. The documents may come from teaching and research institutions in France or abroad, or from public or private research centers.
L'archive ouverte pluridisciplinaire HAL, est destinée au dépôt et à la diffusion de documents scientifiques de niveau recherche, publiés ou non, émanant des établissements d'enseignement et de recherche français ou étrangers, des laboratoires publics ou privés.

\section{(이) $\$$}

Distributed under a Creative Commons Attribution - NonCommercial - NoDerivatives $\mid 4.0$ 
pas de la même époque, mais il est à peu près impossible de distinguer nettement entre eux, d'abord parce que, tracés sur un granit indestructible, ils prennent bien vite le même aspect et le conservent indéfiniment; parce que, d'autre part, répétons-le, il n'y a pas eu solution de continuité entre la période néolithique, la période du fer et la période gallo-romaine, vraisemblablement même elles se sont développées sur un court espace de temps. Dans ce pays pauvre et arriéré l'outillage de pierre, peut-ĉtre d'origine récente, s'est conservé jusqu'à une époque tardive. Toutefois il semble nécessaire de distinguer entre les points où s'accumulent les scories métallurgiques avec habitat gallo-romain et ceux où elles n'apparaissent pas. sans gallo-romain, comme précisément le Collège, Puygaud et Maine du Bos : ceux-ci, qui possèdent l'outillage lithique de beaucoup le plus abondant ef le plus caractéristique, doivent bien, malgré la présence du silex dans les autres, représenter les habitats primitifs.

Pierre et Claude Barrikere.

\section{I,fs foullifes dF Marloux}

(SAôNE-ET-I_oIRE) 1944-1945

Interrompues en $1944^{\prime}$, les fouilles de Marloux reprirent à l'automne de l'annéc suivante et furent exécutées, comme les précédentes, par le propriétaire du champ, M. Claude Bony. Le terrain à explorer étant cultivé, il ne fut possible de mener les travaux que par intermitlence, lorsqu'un certain espace se trouvait Jibre. Au surplus, ils se virent souvent entravés par l'état du sous-sol, oì les recherches ne peuvent êtris conduites avec minutie que dans une période favorable, c'est-àdire quand l'argile houeuse formant la couche archéologian!e n'est ni trop durcic

(1) Voir L. Armand-Calitat, Les fouilles de Marloux, près Mellecey (Saóne-et-Loire) en 19:3, Gallia, III, 1944 , p. 25-41. par la sécheresse, ni rendue liquide par les pluies ${ }^{2}$.

Notre but était double : déterminer autant que possible l'étendue du gisement et mieux connaître le matériel gaulois de cette station.

Nous résolûmes tout d'abord d'explorer le petit chemin (fig. 1,2) situé à l'extrémité ouest du champ, cette région paraissant riche en antiquités. Notre attente ne fut pas déçue. Un pan de mur en pierres grossières, large de $0 \mathrm{~m}$. 90, fut bientôt mis au jour. Les blocs en étaient reliés avec un mortier. Cependant la construction paraît d'origine gauloise, car nous ne recueillîmes dans les déblais que des vestiges pré-romains : une monnaie éduenne en argent, une pièce en potin, imitation des pièces massaliètes au taureau, une fibule à ressort, deux annelets en bronze, un fragment de bouton (?). des poteries grises brisées, des pointes d'amphores, des scories et des culots de fonderie de fer, des tessons de vases ornés de chevrons en creux.

La partie centrale du champ, abandonnée en 1943, fut ensuite attaquée sur $5 \mathrm{~m} .50$ de largeur. Elle nous donna des cubes de mosaïque très nombreux, une cinquantaine au moins, provenant du pavement détruit à la fin du $x^{*} x^{*}$ siècle par les «minages》 de la vigne et qui recouvrait l'établissement gaulois primitif. La tradition nous en avait signalé plusieurs fois l'existence. Mais les précédentes fouilles ne nous avaient permis de retrouver qu'un seul cube, ce qui était un bien faible témoignage. La mise au jour de si nombreux débris lève les derniers doutes qui pouvaient subsister sur la réalité des découvertes antérieures et

(2) Celte nature particulière de terrain est peut-être - remarquons-le en passant - à l'origine du nom de Marloux, Merlotum au $\mathrm{xl}^{\mathrm{e}}$ siècle, rhabillage d'une forme vulgaire Merlo que l'on rencontre dans le département de la Marne (aujourd'hui Merlaut). Dans les deux cas, le prototype, Merlavus ou Merulaous, doit être issu d'un vocable prélatin désignant la boue, d'après les recherches de M. Paul Lebel, spécialiste de l'hydronymie française. 
aussi nous éçlaire sur la qualité de l'ouvrage : nous pouvons dire aujourd'hui qu'il ćtait probablement fort simple, puisque nous n'avons retrouvé que des cubes blancs. Un grand bronze, coupé, assez fruste, de la colonie de vienne et un un morceau de faisselle, percé de trous carrés, trois pièces gauloises, un aiguisoír en pierre verte, des vases ovoides brisés contenant de la cendre, des assiettes, des anses d'amphores. Tout paraît indiquer que nous sommes en présence

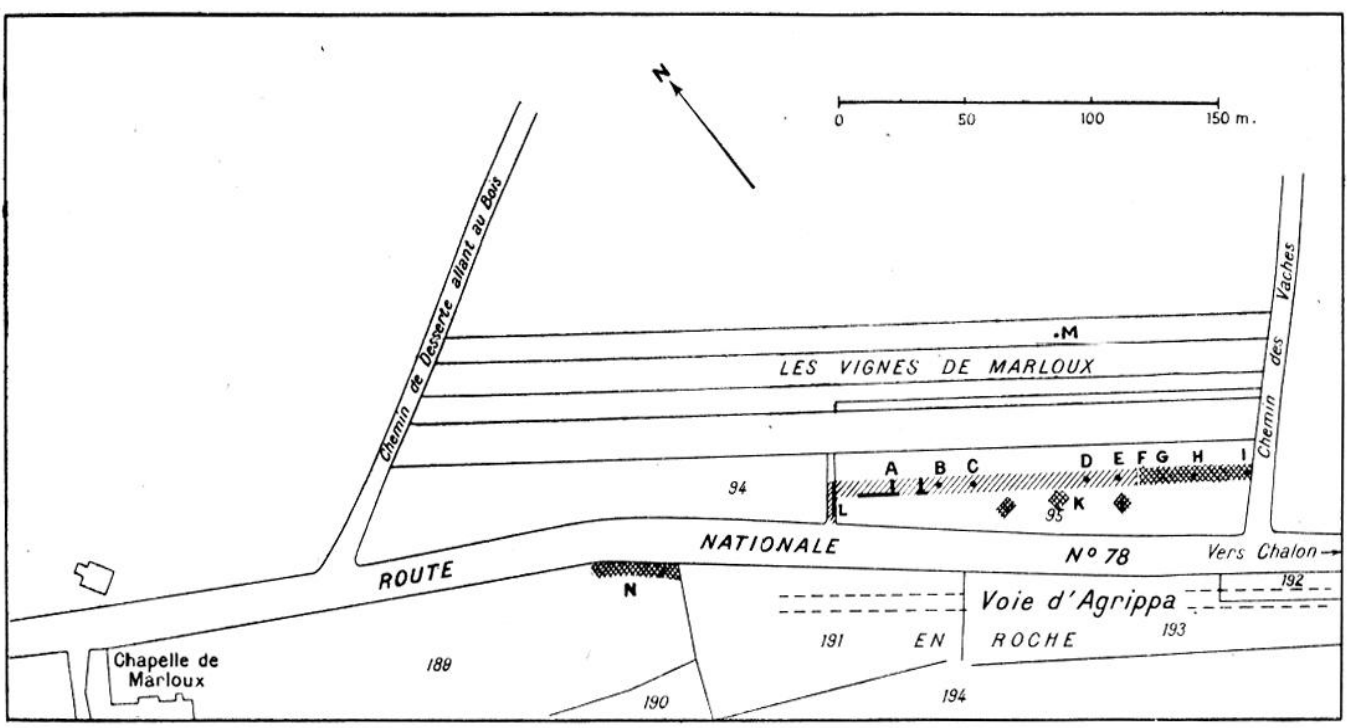

Firi. 1. - Le site de Marlnux.

moyen bronze de Domitien étaient associés à ces débris, ainsi que deux crochets de fer en forme de $T$, un fond de vase en verre, des fibules à charnière, dont unc argentée, paraissant dater du $\mathrm{I}^{\text {er }}$ siècle. quelques menus tessons de poterie sigillée très usés.

A un niveau plus bas, légèrement plus i l'est (fig. 1, H) fut repéré un milieu gaulois, comprenant, dans une accumulation de terre noire visqueuse : une hachette néolithique brisée, trois petits annelets de bronze, des disques en terre cuite, trois morceaux de fer très oxydés, une plaque de fer rongée par la rouille, des anneaux de même métal, des tiges de fer terminées à leur extrémité par une boule (nous croyons qu'il s'agit des débris d'une table ou de chenets gaulois). un fond de petite ampoule en verre bleu. des tessons de poterie grise, notamment d'une sépulture gauloise a incineration. pratiquée sous un foyer et malheureusement assez dispersée par les travaux antiques et modernes effectués at mème endroit.

Près de là (fig. 1) apparut un ens('mble de dalles (fig. 2) posées à plat. Ce sont des blocs calcaires, blancs ou rougeâtres, irréguliers, qui formaient une sorte de petite allée, large seulement de $0 \mathrm{~m}$. 70. Ils étaient placés à même la terre battue, d'ailleurs très serrée et très ferme que l'on rencontre parfois un peu au-dessus de ce gros sable, appelé le cran.

Enfin, à l'extrémité du champ, au bord même du chemin des Vaches (I) fut rencontrée jusqu'à $1 \mathrm{~m} .20$ de profondeur une masse de terre noire, mêlée à une quantité considérable de débris de poteries non vernissées, principalement des assiettes plates ou à bord rabattu inte- 
rieurement, des pointes d'amphores, des ossements d'animaux (défenses de sanglier, dents de cheval), le tout mêlé à une argile durcie et rougie par la flamme.

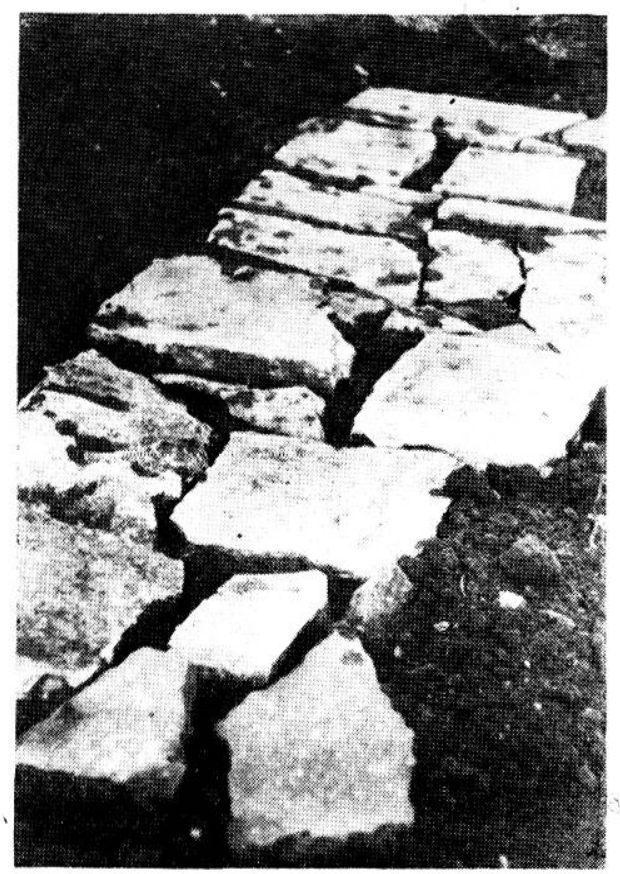

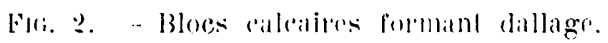

De nombreux et très grands clous de fer forgé semblaient indiquer qu'il s'agit d'une cabane de bois, incendiée, car des fragments ligneux carbonisés adhéraient a certains de ces clavi trabales. La hutte itait probablement de forme ronde et ses parois recouvertes de glaise. Nous avons eu la bonne forlune d'extrairc de la terre noire un vase intact ayant la forme d'une terrine et un autre de profil assez curieux (fig. 10, en bast. D'aillours, ce qui caractérise ce fond de cabane. e'est une accumulation exceptionnelle de débris do poterie el la rarite des restiges métalliques. Seuls un morceau de forces ou ciseaux de fer, deux pièces gau oises et une aiguille de fibule ctaient joints a tant de coramique mutilée.

Le propriétaire de la vigne ayant consenti à sacrifier quelques ceps un peu vicillis, nous arons $\mathrm{pu}$ porter nos invesligations sur la zone située à proximité de la route nationale, en effectuant une série de sondages sporadiques. Ils confirment l'cxistence de murailles assez bien établies, orientées nord-sud et très rajprochées les unes des autres, comme s'il s'agissait d'habitations très cloisonnées. I.es débris recueillis dans les déblais sont tantôt romains, tantôt gaulois et si mélangés que la datation de ces substructions semble malaisée, dans l'ètat actuel de nos recherches. Au reste, a Marloux. il n'y a guère de solution de continuité entre ces deux périodes. Les restiges les plus intéressants recueillis dans cette zone (K) sont une clé en fer à pannetons (en équerre de type romain. et une très belle entréc de serrure en bronze, de

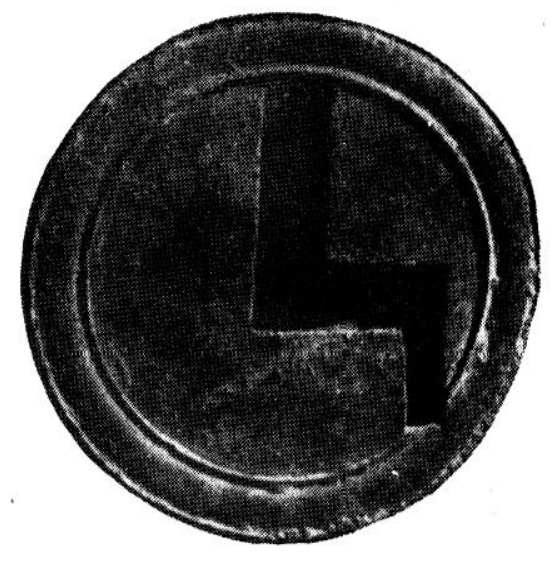

Fui. 3. - Entrée de sirrure en hronm. diamille: 0,08$)$.

forme circulaire, atleignant le diamètre de $0 \mathrm{~m} .08$ (fig. 3). Catte dernière pièce reposait sur un bloc de ciment, vestige probable d'un pavement; elle était associèe à des morceaux de luiles à rebords et à des poteries grises brisées.

Assez près de la voie d'Agrippa, il a été mis au jour une clef en $S$, semblable à celles qui ont été découvertes dans le Chalonnais à Chasscy el a la crangeFrangy (.Mémoires de la société d'Hisloire et d'Archéologie de Chalon, 1869, 
pl. V bis et VIII, fig. 2). Nous la croyons de l'époque mérovingienne et ce qui naus confirme dans cette opinion, c'est qu'une agrafe de vêtement, en bronze ', et un fragment d'ossement humain (tibia) furent peu après rencontrés au même lieu. Les deux pièces doivent provenir d'une sépulture du haut Moyen-Age.

La rectification du tracé de la route nationale $(N)$ nous a permis d'étendre un peu les observations en direction de la Chapelle. Une fois de plus, furent exhumés des tessons de poterie gauloise : dolia à rebord horizontal, coupes à bord rabattu intérieurement, puis des tegulæe, des anses d'amphores et des morceaux de schiste noir. Cependant dans cette zone la couche archéologique préromaine, plus superficielle et moins riche, semble indiquer un habitat moins prolongé.

Pour terminer, nous avons exploré sommairement les terres au nord de la parcelle appartenant à $\mathbf{M}$. Bony. A environ $70 \mathrm{~m}$. de la route nationale, deux pièces en mauvais état de la fin du II' siècle et des morceaux de tuiles à rebords révèlent probablement (en $\mathrm{M}$ ) la destruction de maisons gallo-romaines lors des invasions germaniques.

La station antique de Marloux s'étendait de la sorte assez loin de la voie d'Agrippa en direction du nord. Nous avons vu d'autre part qu'elle atteignait à l'ouest la Chapelle de Marloux et, en sens opposé, au moins le chemin des Vaches qui n'en marquait très probablement pas la limite. On peut juger par là de son importance.

\section{$\stackrel{*}{* *}$}

Le matériel recueilli appelle quelques remarques, surtout en ce qui concerne les œuvres indigènes.

Bronze. - Les annelets de bronze se sont montrés plus fréquents en 1945-1946 qu'au cours de la précédente campagne.

(1) Sur ces agrafes tantôt en fer, tantôt en bronze, voir E. Salin, Le fer à l'époque mérovingienne, Paris, 1943, p. 215.
Le plus grand, en fil mince, atteint $25 \mathrm{~mm}$.; quatre autres, fort solides, mesurent seulement de 14 a $9 \mathrm{~mm}$. On sait que ces petits cercles métalliques abondent au Beuvray (Déchelette, Fouilles, pl. XIV, 35-44).

La récolte des fibules fut au contraire assez faible à Marloux en 1943. Deux exemplaires à ressort, avec arc filiforme, étaient brisés. Trois fibules à charnière restent dépourvues de leur ardillon. L'une d'elles, plaquée d'argent, se rattache au type Aucissa de Morin-Jean (Congrès préhistorique de France, 1910, p. 815, fig. $16, n^{\circ} 5$ ) et doit dater du $\mathrm{I}^{\text {er }}$ siècle. Les autres semblent appartenir à la même époque.

Un objet assez curieux est le disque de $26 \mathrm{~mm}$., malheureusement incomplet. que reproduit notre fig. $6, F$. Il est orné de cercles concentriques et présente une grande analogie avec un bouton découvert dans une hutte gauloise sur le mont Auxois (Pro Alesia, $\mathrm{n}^{\circ} 36$, juin 1909, fig. 116). Il ne semble pas qu'il puisse s'agir d'une fibule.

Nous attirerons spécialement l'attention sur la plaque de serrure (fig. 3), mise au jour près des substructions avoisinant la route romaine. C'est un disque large de 0,08 , d'une exécution très soignée. Cne belle patine verte le recouvre. Il est en bronze coulé et semble avoir été terminé au tour sur sa face externe qui est ornée de moulurations et de filets. Le revers est pourvu de deux tenons de même métal, destinés à fixer l'entrée dans la paroi de la porte. Ces tenons plats, longs de 17 mm., sont eux-mèmes percés d'un trou par lequel devait passer latéralement une petite tige de fer ou un clou dont il resle quelques fragments. Des disques saillants avec entréc de serrure, tout à fait semblables a celui qui nouss occupe, sont figurés sur la porte sculptée dans le marbre de certaines stèles phrygiennes d'AsieMineure (Michon, Mémoires de la Société des Antiquaires de France, t. LXVI, 1906. p. 28, fig. 1 a 3 ). Mais c'est un document 
fort rare dans nos demeures gallo-romaines, beaucoup plus rare que les clés richement ouvragées. Remarquons que celle qui venait s'adapter à cette serrure devait avoir un panneton très compliqué, en double équerre, disposition assez anormale.

Une tige de bronze, brisée et déformée, de section circulaire, mesurant dans son état actuel $75 \mathrm{~mm}$. de longueur, est peut-ètre un vestige de l'anneau permettant de mouvoir le battant de la même porte (Voir Daremberg et Saglio, Dict. des Antiquités, articles Sera et Janua).

Fer. - Quelques débris de fer (fig. 4, $a, b, c, d)$ recueillis groupés dans un milieu gaulois, méritent une mention particulière. Ces tiges terminées par des bul-

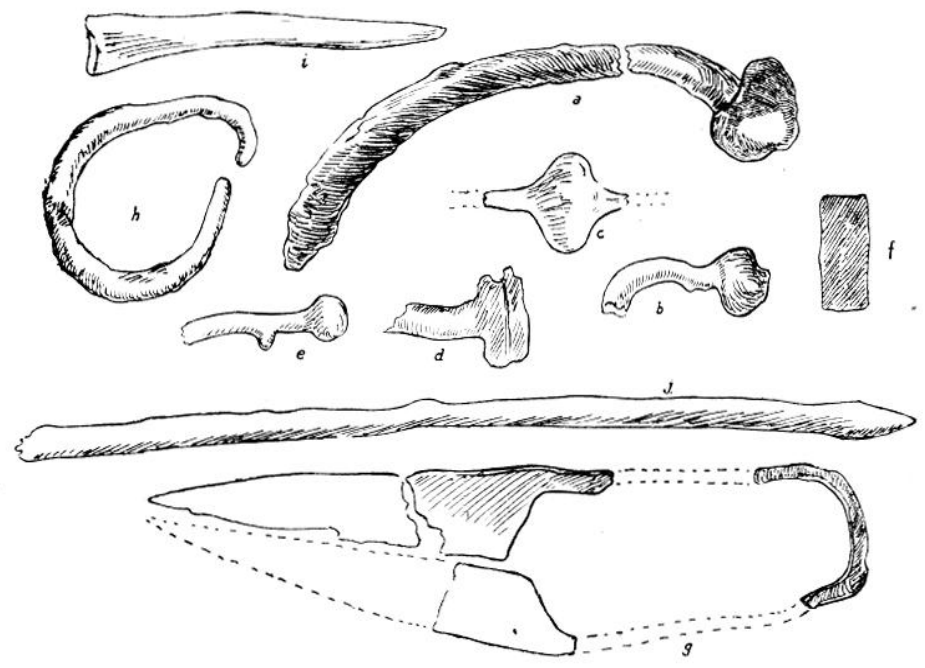

Fici. 4. - Débris d'objets en fer. semblables à celles que nous avons recueillies incomplètes à Marloux (Déchelette, Manuel, p. 1408-1409, fig. 629 et $630)$. On notera qu'un anneau ouvert (h) et que des barres de fer à bout pointu ou aplati, ayant toute l'apparence des tisonniers et des pelles de foyer trouvées au Beuvray (Album Thiollier, pl. XLVII, 3, 4) avoisinaient à Marloux ces mêmes débris $(i, j)$. Un tisonnier à tige torse et qui se termine par une spatule est comparable à ceux de Stradonitz (Bohême) et de la Steinsburg (Saxe).

Les forces (fig. $4, \mathrm{~g}$ ) sont identiques à celles du Beuvray (Album, pl. XLIV, 10). Le lourd poinçon (fig. 5, b) à bout pointu et à tête aplatie par les coups de marteau, devait être destiné à percer des trous.

Le crochet arrondi (fig. 5 , c) se retrouve à Bibracte (Album, pl. XLVI, 20), de même que le clou à double courbe (d') (Voir Album, pl. XLVIII,11).

Les crochets (fig. 5, $\left.g, g^{\prime}, h, h^{\prime}, h^{\prime \prime}\right)$ ressemblent comme forme $\dot{a}$ ceux qui ont été découverts sur l'oppidum éduen (Album, pl. XLVI, $12,13,17)$. Ils nous paraissent cependant trop petits pour avoir été des "gonds de porte».

Les clous (i, $\left.i^{\prime}, i^{\prime \prime}\right)$ sont des clavi trabales de charpentier. Les pièces à équerre ( $\left.f, f^{\prime}\right)$ res-

bes et associées à des morceaux de métal de section quadrangulaire (f) doivent ètre rapprochées des tables et surtout des chenets métalliques découverts dans de nombreuses stations celtiques en Gaule, en Bohême et dans les îles Britanniques, chenets dont les montants sont amortis par des têtes de bovidés, figurées de façon très sommaire. Les cornes bouletées des chenets de Stradonitz (Bohême) et de Welwyn (Hertfordshire) paraissent très tent d'une interprétation incertaine. On peut y avoir soit des débris de clés gauloises en forme d'ancre, type déjà rencontré à Marloux, soit des clous en $T$ d'un profil un peu particulier.

Les six anneaux $(k, 1)$ rappellent par leur exécution grossière ceux du Beuvray (Déchelette, Fouilles, pl. XVI).

Nous devons mentionner aussi une hipposandale brisée, avec talonnière ar- 
mée d'un crochet, et une clé avec panne-

Pierre et matières diverses. - La figure $6, \mathrm{~A}, \mathrm{~B}$ représente une pierre à

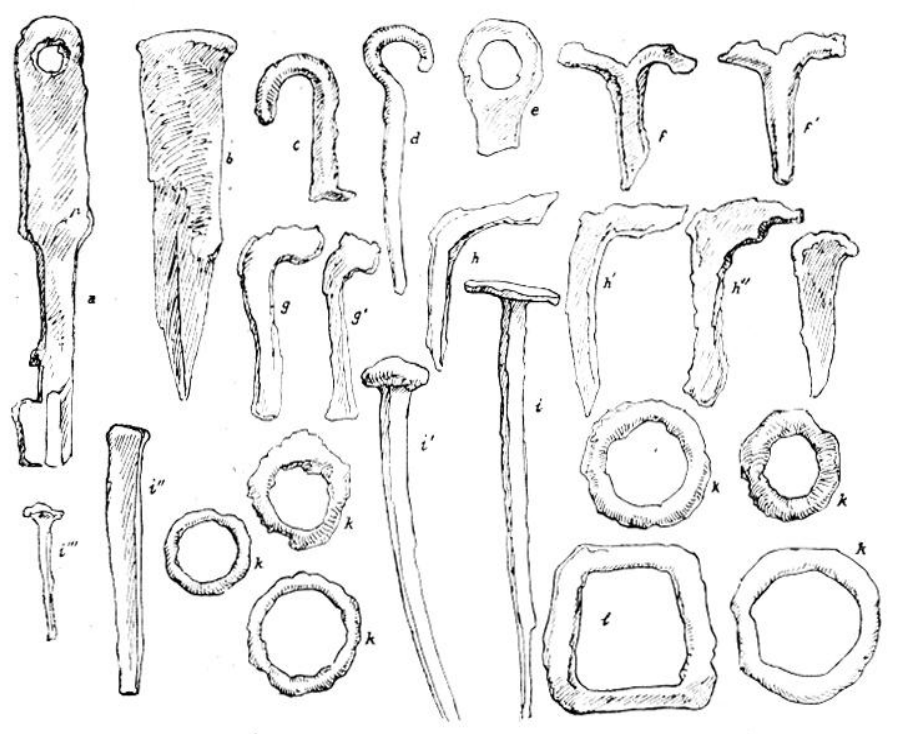

Firi. i. (1)jels divers en Per. tons en équerre (fig. :), a) de type romain.

parois assez epaisses, alteignant jusqu'a $10 \mathrm{~mm}$. L'un d'eux était pourtant décor'é de filets en creux.

Tne statuette en terre blanche, dite de l'Allier, statuette brisée (fig. 6, H) anciennement ol peut-être intentionnellsment, semble provenir d'une sépulture. Flle appartenait à un personnage debout' et qui paraît chaussé.

Une petite ampoule en verre bleu, du diamètre de 18 mm., devait être juinte a une lombe. Il a été recueilli aussi un fond de vase incolore, passé légèrement au jaune, mesurant $50 \mathrm{~mm}$.

Pour ne rien omettre, nous rappellerons la trouvaille - peu après la r'idaction de notre précédent rapport - d'une in-

aiguiser, de coneur verdâtre, assez semblablc a celles dont on se sert encore de nos jours; une autre n'est utilisable que sur une de ses extrémités. Des objets comparables, mais différents, ont éti observés au Beuvray (Album, pl. LVII, 3丂, $37,41)$.

La pierre (fig. 6, (i) paraît avoir été-plụtôt destinée all polissage qu'à l'aiguisage.

Des disques en terre cuite ont été recueilli. au cours des fouilles de 1943. Le plus grand mesure $53 \mathrm{~mm}$. et le pius petit $27 \mathrm{~mm}$. Ce dernier est taille dans un tesson de vase peint, recouvert d'un engobe blanc. Les autres le sont dans des vases de nature grossière et $\dot{a}$
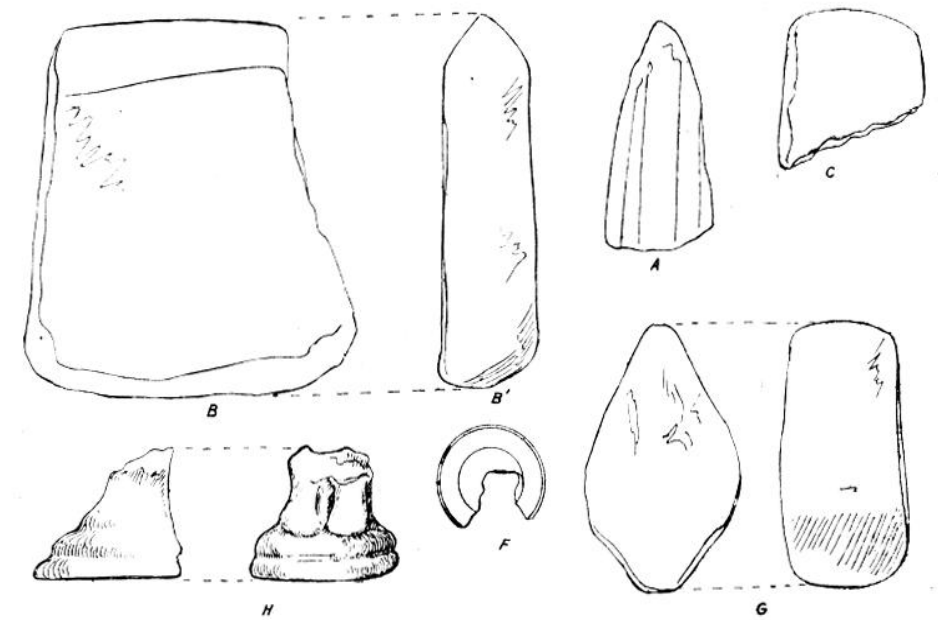

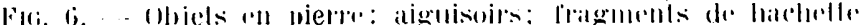

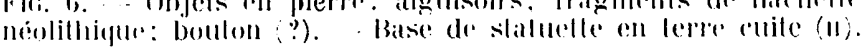

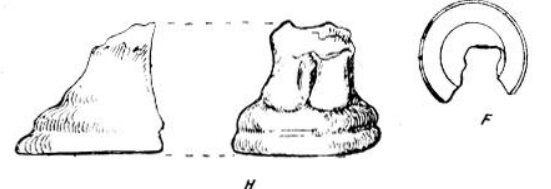

taille en cornaline (fig. 7) dans les deblais de l'ustrinum gaulois, mis au jour 'n décembre 1943. Cette pierre, d'une assez grande finesse, représente un berger, le front ceint d'une couronne ef qui, 
accroupi, joue avec une chèvre retenue par les cornes. Elle est longue de $12 \mathrm{~mm}$.

Vases en terre cuite. - Les recherches de 1945 ont sensiblement étendu nos connaissances relatives à la céramique employée à Marloux pendanl l'épocuc gauIoise.

Outre une partic des types de vases ornés soit à la

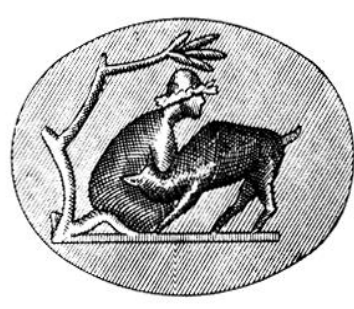

Fili. i. - Intaille en cornaline (agr. 3 fois). pointe ou au lissoir, soit de zones peintes, vases déja rencontrés au cours de la précédente campagne et sur lesquels nous ne reviendrons pas, nous devons noter un certain nombre de motifs nouveaux de décoralion linéaire.

Un tesson (fig. 8, a), en terre noire micacée. présente des zones horizontales de fabriqué à Lezoux dans les ateliers indigènes. Un autre fragment (b) est de même style, mais la terre en est brune. Des morccaux (c et d), décorés à la roulette, doivent être également gaulois. Le décor en points ou en traits verticaux, sur poterie jaune, en terre mal cuite mais assez fine, offre une grande analogie avec certaine céramique de Beuvray (Album, pl. XXXVI, $2,8,11$ ) ou de Chassey (Mém. de la Soc. d'Hist. et d'Arch. de Chalon, 1869; pl. VII, fig. 14). Un vase (e) à engobe noir, de fabrication plus soignée, présente une série de petits dessins ovales ou en spirales, faits à la roulette, qui rappellent, a une moins grande échelle, ceux des poteries de Bibracte (Album, pl. XXXIII, 8, 15) dont le motif est dérivé de la représentation de l'œil (Déchelette, Manuel, p. 1486, fig. 680)

Les grandes jattes à bord rabattu intérieurement, en pâte grise, noire ou rosée, ont le plus souvent la partie horizontale de ce rebord ornée de deux cercles concentriques faits au tour. Cependant les dernières fouilles ont fait apparaître des variétés nouvelles assez intéressantes et de caractère nettement gaulois: sur un exemplaire en effet (fig. 9, a) les lèvres internes et externes présentent des incisions à l'ébauchoir, tandis qu'entre elles se développe un rinceau sinueux. Sur un autre (fig. 9, b) une série d'encoches obtenues par un coup de doigt n'existent qu'à l'intérieur. Sur

chevrons. tracés profondément à la pointe, comme on en a remarqué à $\mathrm{Bi}$ bracte (Album, pl. XXXIV, fig. 11 et 13). Il devait appartenir à un vase ovoïde (Album, pl. XVI), et l'aspect particulier de sa pâte, comparée avec des tessons lédosiens communiqués par M. Charles Fabre, nous donne à penser que nous sommes en présence d'un débris de vase un troisième (fig. 9 , c) la lèvre externe se montrc seule pourvue de cette ondulation. L'un des récipients de cette série offre au-dessous du rebord des hachures obliques, incisions pratiquées à l'ébauchoir et de même facture que celles qui ornent les grands dolia gaulois des ateliers de métallurgistes (Gallia, III, 1944, p. 35, fig. 8), à Marloux et au Beuvray. 
Ln type nouveau à Marloux mais connu à Bibracte (Album, pl. XXI, 6, 8) esl celui des coupes évasées de profils divers. L'une d'elles, celle que reproduit notre

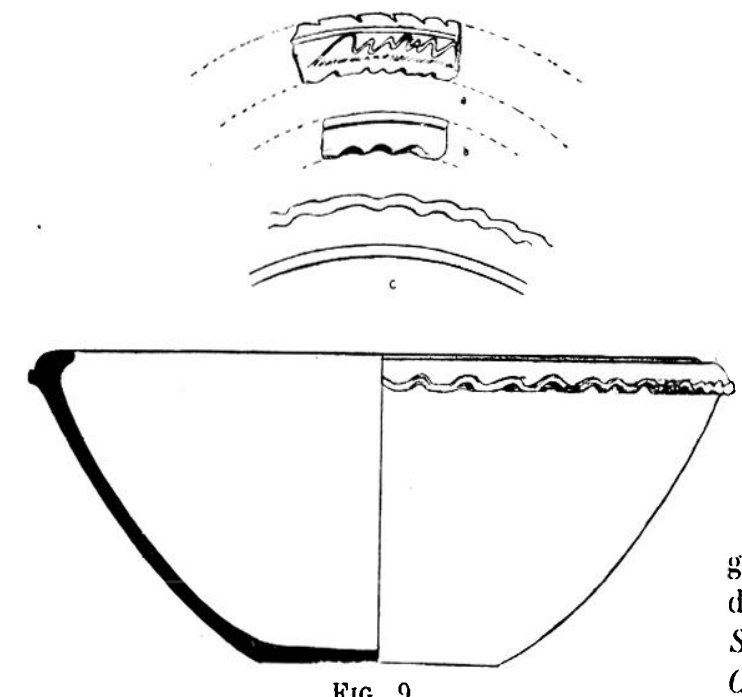

Coupes ornées de cercles concentriques.

figure 9, a, est en terre rouge et engobe noir, fin et bien lissé; cependant la pâte est si mal cuite qu'elle se ramollit dans
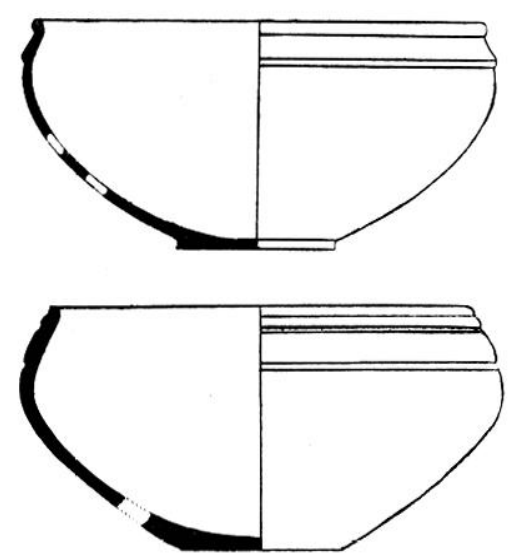

Fis. 10. - Petites coupes.

l'eau; aussi a-t-il èté impossible de recoller les tessons du vase. Un autre exemplaire (fig. 9, b) est en terre violacée et à engobe noir. Un troisième (fig. 9, c) montre une variante du rebord.
Les petites coupes à bord rabattu intérieurement étaient au nombre de cinq, toutes incomplètes d'ailleurs (fig. 10). Certaines offrent un profil cambré, asscz
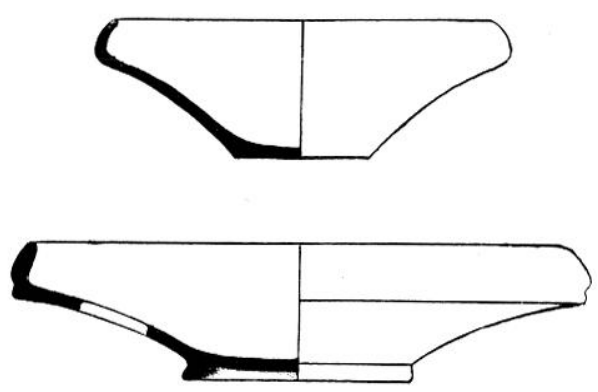

FIG. 11.

Plals pourvus sur les deux faces d'un engobe de couleur mastic.

gracieux, que nous avions déjà rencontré dans le Chalonnais à Granges (Mém. de la Société d'Histoire et d'Archéologie de Chalon, 1940, p. 95, fig. 7).

Le plat (fig. 11) est un modèle intermédiaire entre le type précédent el l'assiette proprement dite. Il est en terre
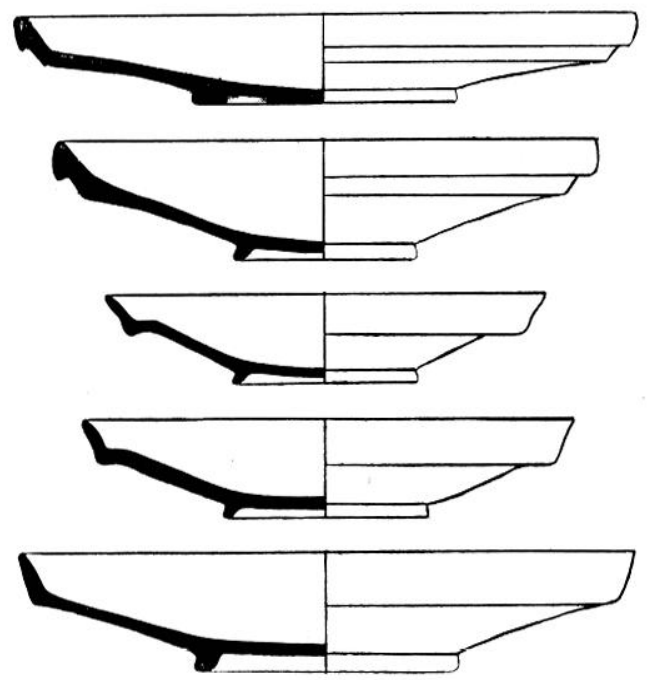

Fili. 12. - Assiettes en lerre grise ou jaunatre.

grise, mais avait été pourvu sur ses deux faces d'un engobe de couleur mastic assez fragile dont il subsiste pourtant des traces très nettes. 
Les assiettes etaient particulièrement nombreuses. Nous avons en effet recueilli les débris d'au moins onze d'entre elles (fig. 12), toutes en terre grise ou jaunâtre. Le profil de leurs rebords est conforme aux dessins donnés par Déchelette pour le Beuvray (Fouilles, pl. XXI, fig. 2, 4, 8). La plupart ont reçu l'engobe mastic que nous retrouvons sur le plat précédemment décrit. L'un des exemplaires présentait sur cet engobe, autour du fond, des lignes concentriques de petits points, très légèrement obtenus à la roulette.

Lne grande terrine large de $175 \mathrm{~mm}$., avec une légère gorge à la partie supérieure, est en terre noire grossière mélangée de gros srains de quartz. L'intérieur avait été pourvu d'un engobe noir très brillant.

Les bols à fond arrondi et dont les lèvres sont renforcées par un rebord plat ont fourni quatre exemplaires brisés, faits de terres diverses, grise, noire, rougeâtre ou jaune, micacéc. Tous avaient des filets horizontaux, de nombre rariable.

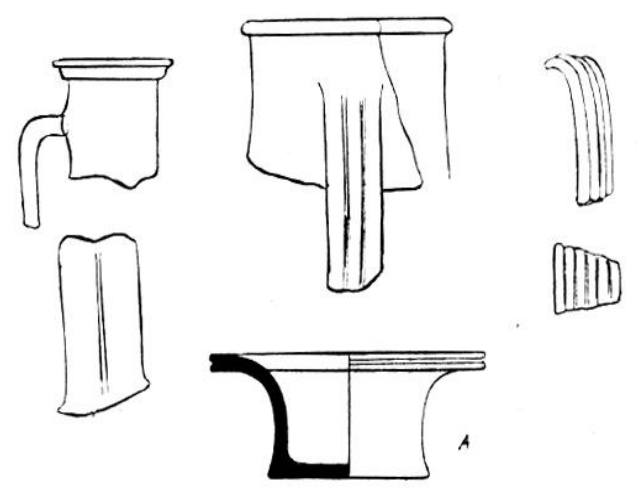

lik. 13. -..- Tilssies, vases à anses, cruches.

Les fouilles de 1945-1946 ont mis au jour un type de vase qui semble rare à l'époque gauloise, du moins en Chalonnais, car nous ne l'avions jamais encore rencontré. C'est une sorte de grande tasse (fig. 13, A) à fond plat, dont le rebord supérieur offre un énorme développement. Trois exemplaires en terre rose ou grise, avec ou sans engobe noir, nous rappellent les récipients du Moyen-Age ou de la période moderne que l'on plaçait sous les faisselles a fromage, provenant des ateliers de Sevrey '.

Les seuls vases à anses sont ou des amphores $\dot{a}$ vin ou des cruches $\dot{a}$ anses droites cannelées. Et sur cinq anses plates (fig. 13) appartenant à des poteries différentes, quatre sont modelées avec cette terre rosée et de cuisson très défeclueuse qui a été usitée au Beuvray pour les récipients de la même série (Album, pl. XXXIX).

Monnaies. - Dix pièces gauloises viennent s'ajouter aux six que nous avions recueillies en 1943 . Deux sont en argent et paraissent éduennes : $1^{\circ}$ buste de femme à gauche avec une légende très effacée; revers, cheval à gauche, au-dessous un dauphin, légende disparue (La Tour, Atlas, XV, 4805); $2^{\circ}$ tête casquée à gauche, revers, cheval à gauche, au-dessous motif indéterminé (peut-être La Tour, 5053).

Les autres pièces gauloises sont des imitations de bronzes massaliètes au taureau. L'une, avec grosse têle d'aspect négroïde, est attribuable à la région de Beaune. Une seconde, avec triple bande, émane probablement des Ségusiaves. Les dernières, fort oxydées, doivent provenir du bassin de la Saône, mais leur détermination est presque impossible.

Onze pièces, toutes en bronze, composent le numéraire romain. Ce sont : un grand bronze coupé de la colonie de Vienne; un Domitien très usé; une Faustine (FAUSTINA AUGUSTA, revers HII.ARITAS, avec l'Allégresse debout); un Commode; un Gallien; cinq Claude le Gothique; un Constantin $\mathrm{I}^{\text {or. }}$. Jes monnaies de Domitien, de Faustine ef de Commode ont beaucoup servi. Par contre, celles de

(1) Sevrey est un village situe tout près de Chalon et dont, au Moyen-Age, presque tous les habitants étaient des potiers spécialisés dans la production d'une céramique noire commune. 
Gallien et de Claude le Gothique sonl soit à fleur de coin, soit très altérées, dirait-on, par la flamme.

$$
* *
$$

Au tolal, les dernières explorations de Marlonx ont confirmé les précédentes. Ėn outre, elles ont permis de micux appricier l'étendue de la station gauloise qui s'étalait sur une longueur d'au moins 400 m. Très prospère vers la fin de l'époque de La Tènc et soumis à l'influence de Bibracte, comme semble !'attester de plus en plus la similitude de la céramique usitée de part el d'autre, ce poste routicr ne fut point abandonne en même temps que ie Beuvray. Ia mélange du matériel gaulois et romain $y$ est au contraire assez remarcquable. Plus fard, lorsque les bâtiments eurent été détruits pa: les Invasions du III $^{\mathbf{e}}$ siècle, des sépultures furent probablement creusées dans les ruines.

\section{Armand-Caldiat. \\ OBJET EN BRONZH DU MUSÉE}

DE ChaLON-SUR-SAONNE

L'objet que nous reproduisons ci-contre et donl nous donnons une description sommaire a été trouvè dans la Saône en 1853 et provient de la collection Jules Chevrier, léguec au Musée de Chalon en 1883 (fig. 1).

Il s'agit d'une hampe en bronze (hatuteur $0 \mathrm{~m} .21$; largeur : $0 \mathrm{~m}$. 32), traverséc par quatre bandes plates et très minces, également en bronze, découpéc's concentriquement et daus la plus basse esl réduite à un simple fragment. Celle qui la surmonte est complète et a conserrí ses cxlrémités qui se terminent par des poinles. A la partie supérieure de la hampe, a l'endroit où elle forme bulbe, sont encastries, sur une des faces, deux tiges fines ayant une direction perpendicutaire. Actuellement brisées, ces petites liges traversaient, de bas en haul. le

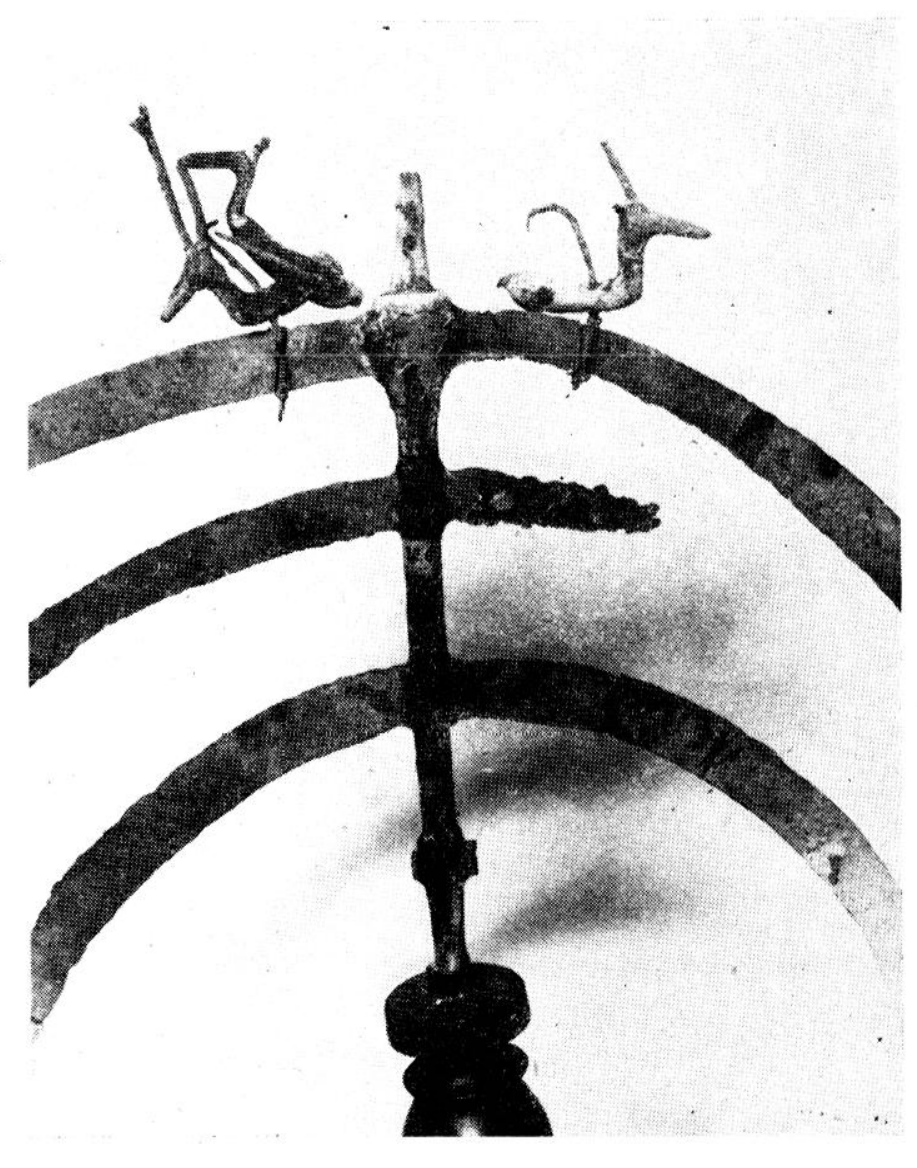

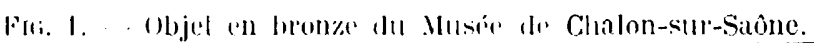

corps dianimaux sommairement ciseles. en ronde bosse, où l'on croil reconnaitre des cervides, dont trois subsistent. II y ('n avait i l'origine au moins quatre, accouplés par deux a l'aide d'une tige qui leur traversait horizontalement les cuisses oul l'arrière-train, de part en part. Le profil aigu donné aux deux extrémités 